\title{
Urologists' Role and Attitude in the Systemic Treatment of Urologic Cancers
}

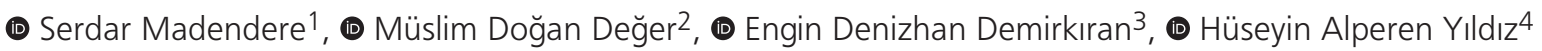 \\ ${ }^{1}$ Gümüşhane State Hospital, Clinic of Urology, Gümüşhane, Turkey \\ 2Edirne Sultan $1^{\text {st }}$ Murat State Hospital, Clinic of Urology, Edirne, Turkey \\ 3Şırnak State Hospital, Clinic of Urology, Şırnak, Turkey \\ ${ }^{4}$ Malazgirt State Hospital, Clinic of Urology, Muş, Turkey
}

\begin{abstract}
Objective: The goal of this study was to investigate Turkish urologists' role and attitude toward the systemic treatment of urologic cancers Materials and Methods: An 18-item survey was e-mailed to 2305 certified Turkish urologists. The survey included questions designed to evaluate urologists' knowledge of chemotherapy procedures and their ability to manage complications, as well as how many of them perform systemic therapies and believe urologists should perform these therapies for urologic cancers.

Results: We evaluated 305 responses. Most urologists (89.6\%) perform hormonotherapy for prostate cancers. Further, the majority refer patients to medical oncologists for chemotherapy. Moreover, $65.9 \%, 70.8 \%$, and $72.1 \%$ of the urologists believe that only medical oncologists should deliver chemotherapy for prostate, bladder, and testicular cancers, respectively. Only $23.9 \%$ of urologists believe they are competent to perform chemotherapy, while $16.7 \%$ believe they are capable of dealing with chemotherapy complications.

Conclusion: Turkish urologists have a lack of performance and interest in administering systemic therapies in urologic cancers. We want to emphasize that urology is more than just surgery. Urologists should be involved in all stages of cancer treatment.

Keywords: Adjuvant treatments, bladder cancer, testicular cancer, prostate cancer, neoadjuvant chemotherapy
\end{abstract}

\section{Introduction}

Urologic cancers accounted for $19.5 \%$ of all cancers diagnosed in the USA in 2019 (1). Among all cancer types, the estimated mortality rate from urologic cancers was $10.9 \%$ (1). The most common urologic cancers were prostate, bladder, kidney, testicular, and penile cancers, respectively (1).

In the diagnosis and management of urologic cancers, urologists are the first point of contact for patients. They perform open, laparoscopic, and robotic surgeries for genitourinary malignancies (2). Systemic therapies may also be necessary at any stage of urologic cancer. In some countries, such as Germany and Japan, urologists administer chemotherapy themselves (3). On the other hand, most urologists in some countries refer patients to medical oncologists due to a lack of expertise in administering chemotherapy and dealing with its complications (4).
The first medical oncology department was established in 1972, and it was recognized as a subspecialty of internal medicine in 1982 in Turkey (5). Urologic oncology is not yet an official subspecialty of urology in Turkey.

Without oncology approval, urologists in Turkey can prescribe bleomycin, etoposide, cisplatin (BEP), neoadjuvant (NA) and adjuvant cisplatin-gemcitabine (CIS-GEM), docetaxel (DCX), goserelin-bicalutamide (GOS-BIC), and zoledronic acid (ZOA). Other systemic therapies, such as abiraterone (ABI), enzalutamide (ENZ), denosumab (DEN), sunitinib (SUN), and pembrolizumab (PEM), require the approval of medical oncologists. All of these systemic therapies are legally permissible to be performed by urologists (6).

In the present study, we conducted a survey among Turkish urologists to obtain a general perspective on the use of systemic therapies for urologic cancers. Of course, diagnosing urologic cancers and performing surgeries are common tasks of

Cite this article as: Madendere S, Değer MD, Demirkıran ED, Yıldız HA. Urologists' Role and Attitude in the Systemic Treatment of Urologic Cancers. Bull Urooncol 2021;20(4):264-269 
urologists. However, we believe that urologists should do more in the treatment of urological cancers, such as administering chemotherapy and other systemic therapies themselves. With this study, we aimed to investigate urologists' role and attitude in the systemic treatment of urologic cancers because we believe there is a deficiency. This study also highlights urologists' interest in and ability to deliver chemotherapy and manage its toxicity. We hope that this study will emphasize that urology is more than just a surgical discipline.

\section{Materials and Methods}

This study included an online survey that was prepared after reviewing relevant articles in the current literature. The survey was constructed using the checklist for reporting results of online E-Surveys (CHERRIES) (7). The survey consists of 18 questions with yes/no or multiple-choices answers about systemic genitourinary cancer treatment from the perspective of urologists. Systemic therapies include hormonotherapy (GOS, $B I C)$, chemotherapy (BEP, CIS, GEM, DCX, ABI, ENZ, SUN, PEM), and supportive therapies (ZOA, DEN). The first section of the survey asks whether urologists perform systemic therapies in their hospitals. The second section includes the opinions of the urologists on whether urologists should perform systemic therapies. In the third section, we investigated respondents' capability to provide and manage complications of systemic treatments. The final question evaluates their understanding of systemic therapies and whether they require oncology approval. Responses indicating that it was necessary for therapies requiring oncology approval or unnecessary for therapies not requiring oncology approval were accepted as true knowledge.

Following a feasibility test with ten respondents, a total of 2305 certified urologists (2223) and urology residents (82) in their final year of training were invited to participate in this study via e-mail. After 4 weeks, reminder e-mails were also sent. Because the study was not based on patient groups, informed consent was not necessary. Between June and October 2020, the survey was available via the online program Google Forms (Alphabet Co., Mountain View, CA). The study was approved by the Local Ethics Committee (2020/10-5).

\section{Statistical Analysis}

Descriptive statistics were used to analyze demographics and practice patterns. The participants were classified according to institute type, academic title, and experience in urology. We demonstrated the proportions of urologists who perform and believe urologists should perform these systemic therapies for genitourinary malignancies. To compare the frequencies, the chi-square test was used. A p-value of $<0.05$ was considered statistically significant. We used the IBM Statistical Package for Social Sciences, version 21.0, to conduct statistical analyses (IBM SPSS Corp., Armonk, NY, USA).

\section{Results}

A total of 317 urologists out of 2305 participated, with a response rate of $13.8 \%$. After excluding 12 incomplete questionnaires from the study, 305 responses were evaluated. The median age of respondents was 36 (27-66). The respondents had experience in urology for a median of 10 (4-39) years. Table 1 depicts the practice patterns and demographics.

Although most urologists perform hormonotherapy to patients with prostate cancer ( $\mathrm{PCa}$ ) by themselves, many refer patients with genitourinary cancers to medical oncologists for chemotherapy. In contrast to hormonotherapy, most respondents believe that oncologists, rather than urologists, should administer chemotherapy (Figure 1).

There was a significant difference that favored academic institutions in performing first-line chemotherapy for testicular cancers $(p=0.006)$. There was no significant difference in performing chemotherapy for other genitourinary cancers between the institutions. Table 2 summarizes the current status of systemic therapies and urologists' attitudes toward them based on institution type and academic title.

A minority of respondents $(n=73,23.9 \%)$ stated that they could perform chemotherapy (Table 3). However, 39 (53.4\%) of those did not feel competent in dealing with chemotherapy complications. It was observed that the rate of urologists who felt capable of managing chemotherapy complications was significantly higher in the group administering chemotherapy $(\mathrm{p}<0.01)$.

Table 4 shows the proportions of urologists who can determine whether the given systemic treatments require the approval of a medical oncologist. Urologists with less than 15 years of experience had a higher percentage of true knowledge about DEN, PEM, and SUN ( $p=0.020, p<0.001, p=0.002)$. However, there was a significant difference in favor of experienced

\begin{tabular}{|l|l|}
\hline \multicolumn{2}{|l|}{ Table 1. Baseline characteristics of 305 respondents } \\
\hline Median age (years) & $36(27-66)$ \\
\hline Academic title & $\mathbf{n}(\%)$ \\
\hline Resident & $76(24.9 \%)$ \\
\hline Specialist & $184(60.3 \%)$ \\
\hline Asst. Professor & $20(6.6 \%)$ \\
\hline Assoc. Professor & $18(5.9 \%)$ \\
\hline Professor & $7(2.3 \%)$ \\
\hline Geographic location & $\mathrm{n}(\%)$ \\
\hline Marmara & $88(28.9 \%)$ \\
\hline Aegean & $43(14.1 \%)$ \\
\hline Central Anatolia & $54(17.7 \%)$ \\
\hline Eastern Anatolia & $18(5.9 \%)$ \\
\hline Southeastern Anatolia & $12(3.9 \%)$ \\
\hline Black Sea & $54(17.7 \%)$ \\
\hline Mediterranean & $36(11.8 \%)$ \\
\hline Experience in urology (years) & $\mathrm{n}(\%)$ \\
\hline $0-5$ & $63(20.7 \%)$ \\
\hline $5-10$ & $110(36.1 \%)$ \\
\hline $10-15$ & $48(15.7 \%)$ \\
\hline $15-20$ & $32(10.5 \%)$ \\
\hline 20 and more & $52(17 \%)$ \\
\hline
\end{tabular}


urologists who believe they can perform chemotherapy $(p=0.018)$. Similarly, urologists in academic hospitals had better proportions of true knowledge about ABI-ENZ, PEM, and SUN $(p=0.045, p=0.041, p=0.040)$. Also, there was a significant

Percentages of departments performing systemic therapies

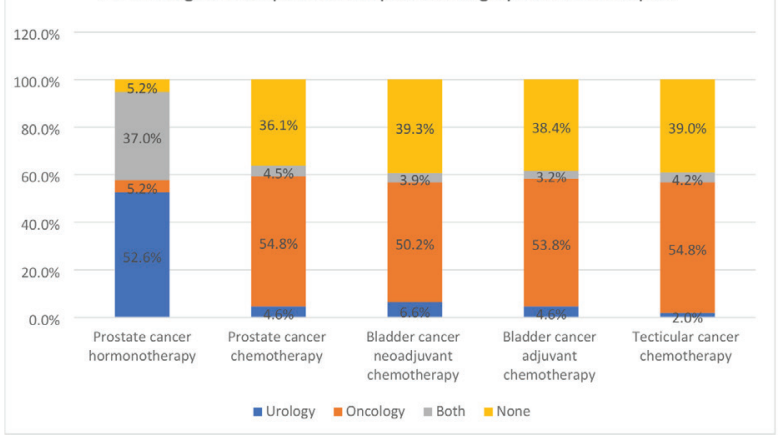

Urologists' opinions about who should perform systemic therapies

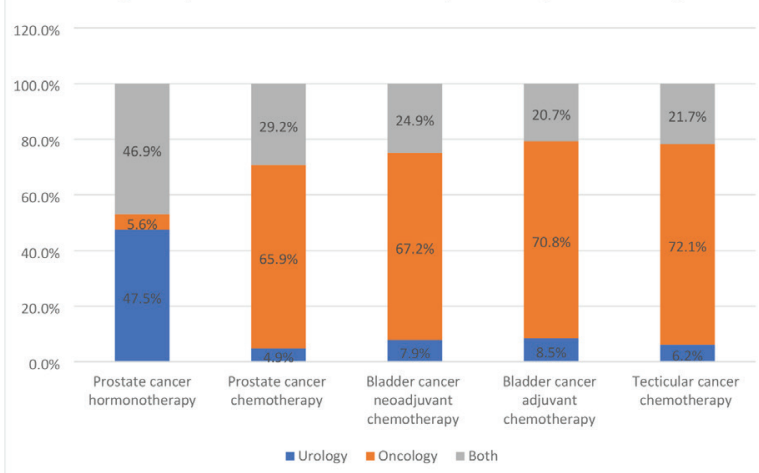

Figure 1. The involvement of urologist in systemic treatments and their perspectives difference favoring this group in terms of feeling capable of managing chemotherapy complications $(p=0.034)$.

\section{Discussion}

The main finding of our study was that most urologists were uninterested in performing chemotherapy and supportive treatment for genitourinary malignancies. But respondents frequently use androgen deprivation therapy (ADT). According to the medical oncology status in Europe Survey, urologists are the primary physicians for screening, diagnosis, and surgery of genitourinary cancers; however, oncologists are on the chemotherapy side (8). There is a similar situation in our study. Inadequate training, lack of interest, and concerns about complications are the most common reasons for this (9). Since the outcomes of our study were similar, we believe the same reasons are valid. We also believe that the key source of motivation in the chemotherapy practice of the urologists is a desire to extend the field of urologic oncology research. Furthermore, in some clinics founded on a similar origin in our country, urologists insist on performing chemotherapy themselves. As a consequence, the residents who were trained in this manner will continue to perform chemotherapy in their future clinics. We think that the common factor among urologists who provide chemotherapy is training under similar environment.

A previous study showed that medical oncology is a wellestablished specialty in many countries, with $77 \%$ of patients receiving chemotherapy for genitourinary cancers in their international study. However, in Japan, only $39.4 \%$ of cancer care hospitals had medical oncology departments (10). In Korea, medical oncologists administered chemotherapy for only $24 \%$ of urological malignancies (11). Urological cancers were treated mostly by urologists, including chemotherapy, in both countries (12). In Turkey, there are 808 medical oncologists working in 240 different institutes. Furthermore, at least 1225

\begin{tabular}{|c|c|c|c|c|c|}
\hline Variables & Types of sys & rapies & & & \\
\hline \multicolumn{6}{|c|}{ Percentages of urologists performing systemic therapies (according to hospital type and academic title) } \\
\hline Hospital type & Pca HT & Рса CT & Bca Neoad. CT & Met. Bca CT & Тса CT \\
\hline Academic $n=148$ & $132(89.2 \%)$ & $15(10.1 \%)$ & $15(10.1 \%)$ & $15(10.1 \%)$ & $15(10.1 \%)$ \\
\hline State $n=106$ & $92(86.8 \%)$ & $10(9.4 \%)$ & $12(11.3 \%)$ & $4(3.8 \%)$ & $2(1.9 \%)$ \\
\hline Private $n=51$ & $49(96.1 \%)$ & $3(5.9 \%)$ & $5(9.8 \%)$ & $5(9.8 \%)$ & $2(3.9 \%)$ \\
\hline Academic title & Pca HT & Рса CT & Bca Neoad. CT & Met. Bca CT & Тса CT \\
\hline Resident $\mathrm{n}=76$ & $71(93.4 \%)$ & $14(18.4 \%)$ & $15(19.7 \%)$ & $14(18.4 \%)$ & $15(19.7 \%)$ \\
\hline Specialist $n=184$ & $163(88.6 \%)$ & $14(7.6 \%)$ & $15(8.2 \%)$ & $8(4.3 \%)$ & $4(2.2 \%)$ \\
\hline Academician $n=45$ & $40(88.9 \%)$ & $0(0 \%)$ & $2(4.4 \%)$ & $2(4.4 \%)$ & $0(0 \%)$ \\
\hline Overall $n=305$ & $274(89.9 \%)$ & $28(9.2 \%)$ & $32(10.5 \%)$ & $24(7.9 \%)$ & $19(6.2 \%)$ \\
\hline \multicolumn{6}{|c|}{ Percentages of urologists thinking urologist should give systemic therapies (according to academic title) } \\
\hline Academic title & Pca HT & Рса CT & Bca Neoad. CT & Met. Bca CT & Тса CT \\
\hline Resident $\mathrm{n}=76$ & $72(94.7 \%)$ & $27(35.6 \%)$ & $26(34.2 \%)$ & $23(30.3 \%)$ & $23(30.3 \%)$ \\
\hline Specialist $\mathrm{n}=184$ & $173(94 \%)$ & $59(32 \%)$ & $60(32.6 \%)$ & $55(29.9 \%)$ & $48(26.1 \%)$ \\
\hline Academician $n=45$ & $43(95.6 \%)$ & $18(40 \%)$ & $14(31.1 \%)$ & $11(24.4 \%)$ & $14(31.1 \%)$ \\
\hline Overall $n=305$ & $288(94.4 \%)$ & $104(34.1 \%)$ & $100(32.8 \%)$ & $89(29.2 \%)$ & 85 (27.9\%) \\
\hline
\end{tabular}


medical oncologists will be needed to provide comprehensive healthcare for cancer patients in Turkey by 2023 (13). We believe that achieving this goal will be difficult. On the other hand, there are 2223 urologists spread across 828 different institutes (14). Urologists should take a more prominent role in the field of chemotherapy by using the advantage of superiority in numbers. Thus, urologists will be able to offer more effective treatment options to patients suffering from urological cancer.

In terms of neoadjuvant chemotherapy (NAC) and adjuvant chemotherapy (AC), most German urology departments

Table 3. Percentages of urologists feeling confident to perform chemotherapy and manage complications

\begin{tabular}{|l|l|l|l|}
\hline Hospital type & Overall & $\begin{array}{l}\text { Feeling } \\
\text { confident to } \\
\text { perform CT }\end{array}$ & $\begin{array}{l}\text { Feeling } \\
\text { capable of } \\
\text { managing CT } \\
\text { complications }\end{array}$ \\
\hline Academic & 148 & $38(25.7 \%)$ & $30(20.3 \%)$ \\
\hline State & 106 & $20(18.9 \%)$ & $11(10.4 \%)$ \\
\hline Private & 51 & $15(29.4 \%)$ & $10(19.6 \%)$ \\
\hline Academic title & 76 & $13(17.1 \%)$ & $11(14.5 \%)$ \\
\hline Resident & 184 & $42(22.8 \%)$ & $25(13.6 \%)$ \\
\hline Specialist & 45 & $18(40 \%)$ & $15(33.3 \%)$ \\
\hline Academician & 305 & $73(23.9 \%)$ & $51(16.7 \%)$ \\
\hline Overall & &
\end{tabular}

administer NAC (81.3\%) and AC (85.7\%) in bladder cancer, which is similar to Japan but differs from many other European countries $(15,16)$. Regardless of whether they work in academic or nonacademic institutions, most Turkish urologists do not administer these therapies and have low interest in NAC and AC. Many of them are unaware that they can prescribe and administer NAC (76.7\%) and AC (74.8\%) without the approval of a medical oncologist. Additionally, referring patients to medical oncologists for NAC can result in a delay in treatment because rescheduling a medical oncology visit can take time. This may cause the optimal time for radical cystectomy (RC) to be delayed. Delays of more than 12 weeks between the diagnosis of bladder cancer and RC can lead to higher mortality and shorter progression-free survival $(17,18,19)$. Urologists can prevent this delay by administering NAC themselves rather than referring patients to oncologists. But, first and foremost, urologists must be interested in and knowledgeable about chemotherapy and its complications.

According to the CHAARTED study, in addition to ADT, chemotherapy is recommended for first-line treatment of metastatic PCa (20). Turkish urologists outperform oncologists in administering hormonotherapy of PCa. However, a minority of urologists perform chemotherapy in PCa with similar percentages to an American study involving the role of the urologist (4). Administering the treatment in two different departments can lead to loss of time and a decrease in treatment effectiveness. If urologists provide both hormonotherapy and chemotherapy

Table 4. Percentages of urologists' true knowledge ${ }^{* *}$ about systemic therapies' approval requirement

\begin{tabular}{|c|c|c|c|c|c|c|c|c|c|c|}
\hline \multirow{2}{*}{$\begin{array}{l}\text { Variables } \\
\text { Hospital type }\end{array}$} & \multicolumn{6}{|c|}{ Therapies not requiring oncology approval } & \multicolumn{4}{|c|}{ Therapies requiring oncology approval } \\
\hline & BEP & $\begin{array}{l}\text { Neoad. } \\
\text { C-G }\end{array}$ & Adj. C-G & DOC & G-B & ZA & $\begin{array}{l}\mathrm{ABI}- \\
\mathrm{ENZ}\end{array}$ & DEN & SUN & PEM \\
\hline Academic $n=148$ & $30.4 \%$ & $24.3 \%$ & $23.6 \%$ & $33.1 \%$ & $71.6 \%$ & $76.4 \%$ & $66.9 \%$ & $59.5 \%$ & $83.8 \%$ & $83.1 \%$ \\
\hline State $n=106$ & $38.7 \%$ & $22.6 \%$ & $25.5 \%$ & $37.7 \%$ & $80.2 \%$ & $79.2 \%$ & $58.5 \%$ & $68.9 \%$ & $71.7 \%$ & $75.5 \%$ \\
\hline Private $n=51$ & $25.5 \%$ & $21.6 \%$ & $29.4 \%$ & $41.2 \%$ & $78.4 \%$ & $84.3 \%$ & $49.1 \%$ & $56.9 \%$ & $64.7 \%$ & $66.7 \%$ \\
\hline \multicolumn{11}{|l|}{ Academic title } \\
\hline Resident $n=76$ & $30.3 \%$ & $22.4 \%$ & $23.7 \%$ & $34.2 \%$ & $72.4 \%$ & $76.3 \%$ & $64.5 \%$ & $67.1 \%$ & $80.3 \%$ & $78.9 \%$ \\
\hline Specialist $n=184$ & $34.2 \%$ & $22.8 \%$ & $26.6 \%$ & $38.6 \%$ & $77.2 \%$ & $79.3 \%$ & $60.3 \%$ & $71.2 \%$ & $76.1 \%$ & $78.3 \%$ \\
\hline Academician $n=45$ & $28.9 \%$ & $26.7 \%$ & $22.2 \%$ & $28.9 \%$ & $75.5 \%$ & $80 \%$ & $57.8 \%$ & $66.7 \%$ & $71.1 \%$ & $73.3 \%$ \\
\hline \multicolumn{11}{|l|}{ Experience } \\
\hline $0-5$ years $n=63$ & $31.7 \%$ & $25.4 \%$ & $25.4 \%$ & $34.9 \%$ & $68.3 \%$ & $81 \%$ & $60.3 \%$ & $66.7 \%$ & $76.2 \%$ & $74.6 \%$ \\
\hline $5-10$ years $n=110$ & $32.7 \%$ & $24.5 \%$ & $27.3 \%$ & $35.5 \%$ & $80 \%$ & $76.4 \%$ & $69.1 \%$ & $75.5 \%$ & $85.5 \%$ & $89.1 \%$ \\
\hline $10-15$ years $n=48$ & $22.9 \%$ & $20.8 \%$ & $25 \%$ & $41.7 \%$ & $75 \%$ & $81.3 \%$ & $58.3 \%$ & $77.1 \%$ & $77.1 \%$ & $81.3 \%$ \\
\hline $15-20$ years $n=32$ & $28.1 \%$ & $25 \%$ & $18.8 \%$ & $34.4 \%$ & $78.1 \%$ & $84.4 \%$ & $43.8 \%$ & $56.3 \%$ & $59.4 \%$ & $59.4 \%$ \\
\hline$>20$ years $n=52$ & $44.2 \%$ & $19.2 \%$ & $25 \%$ & $34.6 \%$ & $75 \%$ & $75 \%$ & $57.7 \%$ & $61.5 \%$ & $67.3 \%$ & $65.4 \%$ \\
\hline Overall $n=305$ & $32.5 \%$ & $23.3 \%$ & $25.2 \%$ & $36.1 \%$ & $75.7 \%$ & $78.7 \%$ & $61 \%$ & $69.5 \%$ & $76.4 \%$ & $77.7 \%$ \\
\hline $\begin{array}{l}\text { Feeling confident to perform } \\
\text { CT } n=73\end{array}$ & $52.1 \%$ & $43.8 \%$ & $42.5 \%$ & $56.2 \%$ & $75.3 \%$ & $80.8 \%$ & $49.3 \%$ & $63 \%$ & $71.2 \%$ & $71.2 \%$ \\
\hline $\begin{array}{l}\text { Feeling capable of managing } \mathrm{CT} \\
\text { complications } n=51\end{array}$ & $49 \%$ & $39.2 \%$ & $37.3 \%$ & $52.9 \%$ & $78.4 \%$ & $88.2 \%$ & $49 \%$ & $60.8 \%$ & $74.5 \%$ & $70.6 \%$ \\
\hline \multicolumn{11}{|c|}{$\begin{array}{l}\text { *ABI-ENZ: Abiraterone-enzalutamide, Neoadj. C-G: Neoadjuvant cisplatin-gemcitabine, Adj C-G: Adjuvant cisplatin-gemcitabine, DEN: Denosumab, SUN: Sunitinib, PEM: } \\
\text { Pembrolizumab, G-B: Goserelin-bicalutamide, DOC: Docetaxel, ZA: Zoledronic acid, BEP: Bleomycin-etoposide-cisplatin } \\
\text { **The first six columns of the table are the percentages of the respondents who state that medical oncology approval is not mandatory. The last four columns of the table } \\
\text { are the percentages of the respondents who state that medical oncology approval is mandatory }\end{array}$} \\
\hline
\end{tabular}


themselves, these risks can be minimized. According to a previous study involving characteristics of physicians treating castration-resistant prostate cancer (CRPC) by country, urologists treated CRPC in Japan, Germany, the USA, the UK, and France with percentages of $98.7 \%, 92.9 \%, 74 \%, 73.4 \%$, and $56.7 \%$, respectively (3). On the other hand, another study in the USA found that oncologists used chemotherapy more frequently than urologists for CRPC (21). In both studies, higher percentages of urologists believe that urologists should perform chemotherapy in PCa than the percentages of urologists who actually perform it. Nonetheless, the majority of urologists think that chemotherapy is a subject for medical oncology. Therefore, the training and perspective of urologists should be investigated. Previous research has shown that urologists can quickly adapt to other systemic treatments for PCa, as they did for hormonotherapy $(22,23)$.

For the first decade after introducing BEP chemotherapy for testicular cancer, urologists administered these therapies in many hospitals until the drawbacks of drug toxicity were discovered. Similar reservations were expressed regarding the systemic treatment of kidney tumors (24). Nowadays, more than $70 \%$ of Turkish urologists say they would refer testicular cancer patients to medical oncologists for even first-line chemotherapy because the majority do not believe they are capable of managing chemotherapy complications (83.3\%). Furthermore, $70 \%$ of respondents think that medical oncology approval is necessary for BEP treatment, although it is not. Even many urologists who are confident in their ability to perform chemotherapy had low percentages of true knowledge about oncology approval requirements for certain types of systemic therapies.

For many years, urologists have used systemic therapies such as ADT, intravesical chemotherapy, and bacillus Calmette-Guerin therapy. These therapies can have life-threatening side effects (25). Nowadays, many urologists can manage complications of these therapies through training in residency and experience over the years. With education, practice, and a strong interest, similar competency can occur over time for intravenous (iv) chemotherapy of urologic cancers.

If urologic oncologists want to become comprehensive care providers for genitourinary cancers, they should be active beyond the operating room. Performing all systemic therapies themselves would provide increased patient satisfaction and professional satisfaction of urologists, avoid delays in treatment, and add a significant field of research in urology (9). Additionally, we hope that urologic oncology will be recognized as an official subspecialty of urology, allowing urologic oncologists to provide comprehensive healthcare to patients.

\section{Study Limitations}

Our study is the first nationwide research that shows the current status of systemic urologic cancer treatment among Turkish urologists. There were several limitations to this study. First, as with any survey study, there is a possible selection bias. The respondents may or may not be interested in the systemic treatment of urologic cancers. Due to demographic bias, the findings cannot be expanded. The overall response rate represents a small proportion of all urologists, but it reveals the general situation. Academic participation rate in the survey was lower than that of specialists and residents. Another point to consider is that cancer treatment is rapidly evolving due to the newly-introduced drugs. The systemic therapies mentioned in this study may become obsolete in the near future. Nevertheless, we found a lack of interest in current medical treatments for urologic cancers among urologists.

\section{Conclusions}

Turkish urologists have a lack of performance and interest in administering systemic therapies for urologic cancers. Training in urologic oncology should include not only operations but also systemic treatments. Currently, treatment of genitourinary cancers requires a multidisciplinary approach between urologists, medical oncologists, radiation oncologists, and other specialists. Urologists, on the other hand, should be aware that they are the primary physicians for urologic cancers and are legally competent to manage urologic oncology patients at all stages.

\section{Acknowledgements}

Publication: The results of the study were not published in full or in part in form of abstracts.

Contribution: There is not any contributors who may not be listed as authors.

Conflict of Interest: No conflict of interest was declared by the authors.

Financial Disclosure: The authors declared that this study received no financial support.

\section{Ethics}

Ethics Committee Approval: The study was approved by the Local Ethics Committee (2020/10-5).

Informed Consent: Because the study was not based on patient groups, informed consent was not necessary.

Peer-review: Externally peer-reviewed.

\section{Authorship Contributions}

Concept: S.M., M.D.D., E.D.D., H.A.Y., Design: S.M., M.D.D., E.D.D., H.A.Y., Data Collection or Processing: S.M., M.D.D., E.D.D., H.A.Y., Analysis or Interpretation: S.M., M.D.D., E.D.D., H.A.Y., Literature Search: S.M., M.D.D., E.D.D., H.A.Y., Writing: S.M., M.D.D., E.D.D., H.A.Y.

\section{References}

1. Siegel RL, Miller KD, Jemal A. Cancer statistics, 2019. CA Cancer J Clin. 2019;69:7-34.

2. Mickelson J], Macneily AE. Surgical case volume in canadian urology residency: a comparison of trends in open and minimally invasive surgical experience. J Endourol 2011;25:1063-1067.

3. Shah R, Botteman M, Waldeck R. Treatment characteristics for nonmetastatic castration-resistant prostate cancer in the United States, Europe and Japan. Futur Oncol 2019;15:4069-4081.

4. Crawford ED. The role of the urologist in treating patients with hormone-refractory prostate cancer. Rev Urol 2003;5(Suppl 2):S48-S52. 
5. Medical oncology establishment in Turkey. Last Accessed Date: 12.11.2020. Available from: https://www.tip.hacettepe.edu.tr/ bolumler/ichast.php

6. Chemotheapy administration procedures in Turkish health system. Last Accessed Date: 07.11.2020. Available from: https:// www.resmigazete.gov.tr/eskiler/2007/05/20070525M1-3. htm\#_Toc167681848

7. Eysenbach G. Improving the quality of web surveys: the Checklist for Reporting Results of Internet E-Surveys (CHERRIES). J Med Internet Res 2004;6:e34. doi: 10.2196/jmir.6.3.e34.

8. European Society for Medical Oncology Phase3 Study. Last Accessed Date: 12.11.2020. Available from: https://www.esmo.org/content/ download/8358/170037/file/2008-ESMO-MOSES-Phaselll.pdf

9. Penson DF, Lange PH. Systemic therapy and the urologic oncologist: a unique opportunity for the specialty to provide comprehensive care that ultimately benefits the patient. Urol Oncol 2012;30(Suppl 4):S2-S4.

10. Komiya T, Mackay $C B$, Chalise P. Status of oncologic specialties: global survey of physicians treating cancer. Int J Clin Oncol 2017;22:237-243.

11. Akaza H. Report from the 1st Japanese Urological AssociationJapanese Society of Medical Oncology joint conference, 2006: "A step towards better collaboration between urologists and medical oncologists." Int J Urol 2007; 14:375-383.

12. Saijo N, Miki T, Kubota Y, et al. Report from the second Japanese Urological Association-Japanese Society of Medical Oncology joint conference, 2007: "Diagnosis and treatment of urological malignant tumors: How can we promote subspecialists?" Int J Urol 2008;15:389-393.

13. Turkish Oncology Services Handbook, May 2011. Last Accessed Date: 21.11.2020. Available from: https://www.kanser.org/saglik/ userfiles/file/11Mayis2011/turkiye_onkoloji_hizmetleri_kitapcik.pdf

14. The Ministry of Health of Turkey Health Statistics Yearbook. 2018. Last Accessed Date: 21.11.2020. Available from: https://dosyasb. saglik.gov.tr/Eklenti/36164,siy2018en2pdf.pdf?0
15. Dogan S, Hennig M, Frank T, et al. Acceptance of adjuvant and neoadjuvant chemotherapy in muscle-Invasive bladder cancer in Germany: a survey of current practice. Urol Int 2018;101:25-30.

16. Anan G, Hatakeyama S, Fujita N, et al. Trends in neoadjuvant chemotherapy use and oncological outcomes for muscleinvasive bladder cancer in Japan: a multicenter study. Oncotarget 2017;8:86130-86142.

17. Değer MD, Çelik S, Yıldız A, et al. Can we perform frozen section instead of repeat transurethral resection in bladder cancer? Urol Oncol 2021;39:237.e15-237.e20.

18. Kuş T, Aktaş G. Maintenance treatment with gemcitabine have a promising activity on metastatic bladder cancer survival. Turk J Urol 2017;43:273-278.

19. Chu AT, Holt SK, Wright JL, et al. Delays in radical cystectomy for muscle-invasive bladder cancer. Cancer 2019;125:2011-2017.

20. Kyriakopoulos CE, Chen YH, Carducci MA, et al. Chemohormonal therapy in metastatic hormone-sensitive prostate cancer: long-term survival analysis of the randomized phase III E3805 chaarted trial. J Clin Oncol 2018;36:1080-1087.

21. Engel-Nitz NM, Alemayehu B, Parry D, Nathan F. Differences in treatment patterns among patients with castration-resistant prostate cancer treated by oncologists versus urologists in a US managed care population. Cancer Manag Res 2011;3:233-245.

22. Caram MEV, Kaufman SR, Modi PK, et al. Adoption of abiraterone and enzalutamide by urologists. Urology 2019;131:176-183.

23. Shahinian VB, Kuo YF, Freeman JL, Goodwin JS. Determinants of androgen deprivation therapy use for prostate cancer: Role of the urologist. J Natl Cancer Inst 2006;98:839-845.

24. Klotz L. Sunitinib, sorafenib and other systemic noncytotoxic kidney cancer therapies can and should be administered by urologists. J Can Urol Assoc 2007;1(Suppl 2):69-70.

25. Tareen B, Taneja S. Complications of intravesical therapy. In: Taneja S, eds. Complications of Urologic Surgery: Prevention and Management. 4th ed. Philadelphia: Elsevier Saunders; 2009. p. 95-102. 